The quantitative reverse transcriptase PCR (qRT-PCR) was used to analyse the expression of DUXAP8 in 50 PC tissues and correspond adjacent normal tissues and three PC cell lines. Loss of function approaches was used to investigate the biological role of DUXAP8 both in vitro and in vivo. Bioinformatics analysis followed by qRT-PCR was performed to identify the putative targets of DUXAP8, which were further verified by RNA immunoprecipitation (RIP), Chromatin immunoprecipitation (ChIP), rescue experiments and western blotting assays.

Results In this study, we analysed of GEO RNA sequencing data and other publicly available microarray data. We found that a pseudogene, DUXAP8, which expression was significantly up-regulated in PC tissues compared to adjacent normal tissues. Furthermore, qRT-PCR results verified that DUXAP8 is over-expressed in PC tissues. In vitro and in vivo assays of DUXAP8 alterations revealed a complexly integrated phenotype affecting cell growth and apoptosis. Mechanistically, DUXAP8 repressed underlying target gene CDKN1A and KLF2 transcription through binding to histone methyltransferase EZH2 and histone demethylase LSD1.

Conclusions DUXAP8 is significantly up-regulated in PC tissues compared with adjacent normal tissues, suggesting that the ectopic expression of DUXAP8 was related to the tumorigenesis of PC. Mechanistic investigations showed that DUXAP8 could repress the tumour suppressors CDKN1A and KLF2 by recruiting EZH2 and LSD1, thereby affecting cell proliferation and apoptosis in PC. Taken together, our findings indicated that the pseudogene DUXAP8 might act as an oncogene in PC by silencing CDKN1A and KLF2 transcription by binding with EZH2 and LSD1, which may serve as a new therapeutic target in pancreatic cancer.

\section{IDDF2018-ABS-0157 A REVISIT ON PERSONALISED CRITICAL VARIANCE OF GUT MICROBIOTA DURING THE OCCURRENCE OF TYPE L DIABETES}

Tao Zeng*, Lu Wang, Xiangtian Yu. Key Laboratory of Systems Biology, Institute of Biochemistry and Cell Biology, Chinese Academy of Sciences, Shanghai, China

\subsection{6/gutjnl-2018-IDDFabstracts.17}

Background As well-known, the gut microbiota is associated with many human complex diseases. The change of microbiota community is observed in diseased individuals. However, the disorder of gut microbiota during disease occurrence is still unclear, and especially the pre-disease or early-disease signal on individual gut microbiota requires systematical researches.

Methods Different from conventional studies on the differential average abundance of gut microbiota between normal and diseased samples, we investigate the variance of the abundance of gut microbiota on consecutive samples from a healthy state to pathogen state for each individual person because the change of microbiota abundance variance would be a critical signal of the biological dynamical system. Edge-network analysis (ENA) is our proposed newly computational approach to analyse the network of associations rather than the network of base variables; especially it can consider the change of variance and covariance simultaneously. Thus we used ENA to integratively analyse the metagenomics data of total 15 individuals from three cohorts in public domain, and each person in the cohorts would have multiple faeces samples for more than one year.

Results Six individuals keep healthy, and six individuals occur seroconversion, and three individuals occur seroconversion along with final Type 1 Diabetes. Focused on the
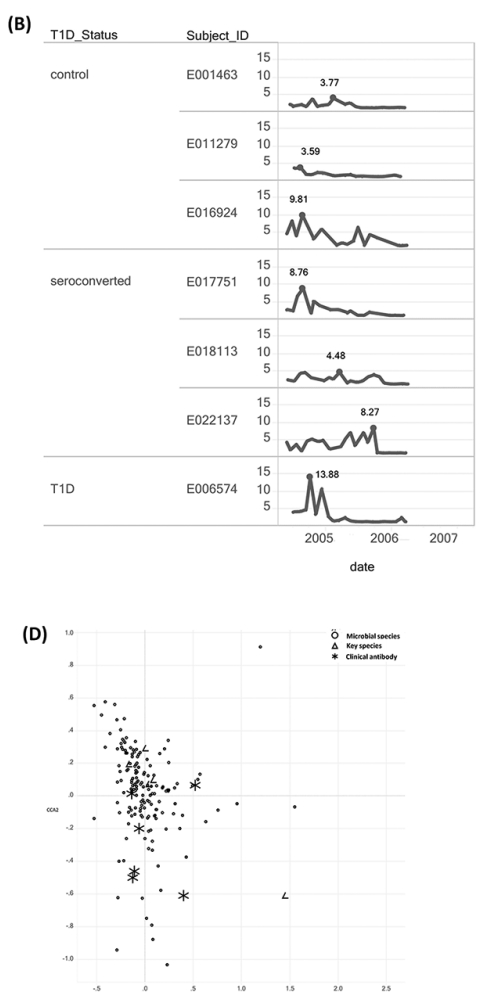

Abstract IDDF2018-ABS-0157 Figure 1 (A) the summary of microbiota abundance for different groups of samples; (B) the personalised index of abundance variance change based on dynamic network biomarker theory; (C) the ROC and AUC of classification; (D) The CCA among microbiota and clinical indices 
seroconversion, a key stage to $\mathrm{T} 1 \mathrm{D}$, the conventional analysis found some microbiota with changed abundance after the occurrence of seroconversion (figure 1a). Meanwhile, our analysis further show that a lot of microbiota actually have great abundance variance change before seroconversion (figure $1 \mathrm{~b}$ ), which can even be efficient features to classify the healthy and seroconversion individuals with about $80 \%$ accuracy (figure 1c). More importantly, this microbiota with critical abundance variance as key species is also associated with T1D clinical antibody or even some new (figure 1d).

Conclusions Dissimilar to common biomarkers like a clinical antibody, the individual specific signatures, e.g. variance of gut microbiota abundance, would be an alternative approach for personalised pre-disease or early-disease diagnosis.

\section{IDDF2018-ABS-0161 GUT MICROBIOME ACROSS STAGES OF HBV INFECTION}

Zhangran Chen*, Yurou Xie, Chuanxing Xiao, Xiang Zhang, Jianlin Ren. Institute for Microbial Ecology, Medical College of Xiamen University, China

\subsection{6/gutjnl-2018-IDDFabstracts. 18}

Background Increasing evidence suggests that the gut microbiota has evolved as a new important player in the pathogenesis of hepatitis B virus-induced chronic liver disease, including chronic hepatitis, cirrhosis. However, the composition and structure alteration of the gut microbiota associated with the stage and progression of $\mathrm{HBV}$ infection remains unknown. Hence, the aim of the study is to elucidate the microbial influence which contributes to the microbial shift of $\mathrm{HBV}$ patients in different stages.

Methods All subjects enrolled had clinic test performed in the Zhongshan Hospital, Xiamen University. A total of 62 subjects were finally selected for inclusion in this study, including 7 healthy individuals, $13 \mathrm{HBeAg}$ negative chronic $\mathrm{HBV}$ infection patients (HNCHI), $6 \mathrm{HBeAg}$ negative chronic hepatitis B patients (HNCHB), $5 \mathrm{HBeAg}$ positive chronic hepatitis $\mathrm{B}$ patients (HPCHB) and 31 decompensated cirrhosis after hepatitis $\mathrm{B}$ patients (DCAHB) matched in age, gender and body mass index (BMI). Stools from all subjects were collected to extract DNA, then for $16 \mathrm{~S}$ rDNA high-throughput sequencing and further compared by the bacterial diversity, community and structure, differential taxa analysis.

Results DCAHB patients showed decreased gut bacterial diversity and more unique OTU numbers compared to the other four groups, suggesting that DCAHB is distinctive, which could further be confirmed by the PCA analysis. In phyla level, the relative abundance of Fusobacteria and Proteobacteria increased while Firmicutes content decreased from the health, HNCHI, HNCHB, HPCHB to DCAHB group. In genus level, the high level of Streptococcus, Veillonella, Enterobacteriaceae, Lactobacillus and Haemophilus, while decreased Clostridiaceae, Phascolarctobacterium, Coprococcus and Ruminococcaceae in DCAHB conform the disease progression state. In addition, LEfSe analysis reveals that Bacteroides fragilis, Bacteroides eggerthii, Veillonella dispar, Streptococcus anginosus, Corprococcus eutactus and Akkermansia muciniphila could serve as target microorganism for the further investigation.

Conclusions These compositional and structural changes in the gut microbiota in varied stage HBV patients suggest the potential contributions of gut microbiota to the progression of $\mathrm{HBV}$ infection and thus provide new insight into gut microbiota-targeted interventions to improve the prognosis of this disease.

\section{IDDF2018-ABS-0162 ALTERED GUT MICROBIOTA IN EXPERIMENTAL MOUSE MODELS OF NON- ALCOHOLIC FATTY LIVER DISEASE}

Bangzhou Zhang*, Lina Fan, Chuanxing Xiao, Jianlin Ren. Department of Gastroenterology, Zhongshan Hospital Affiliated to Xiamen University, China

\subsection{6/gutjnl-2018-IDDFabstracts. 19}

Background Non-alcoholic fatty liver disease (NAFLD) has become the most common liver disease worldwide and is thought to be strongly associated with gut microbiota. Several diet models were therefore built in mice to try to clarify the molecular mechanisms. However, how and to what extent these diet models alter the composition of the gut microbiota have not yet been clearly elucidated.

Methods In this study, we developed three mouse models of NAFLD using methionine-choline-deficient (MCD) diet, highfat (HF) diet, and choline-deficient- high-fat (CD-HF) diet, evaluated the severity of steatohepatitis and sequenced the faecal bacteria by targeting $16 \mathrm{~S}$ V4-V6 regions on Illumina MiSeq using PE 300 reagents.

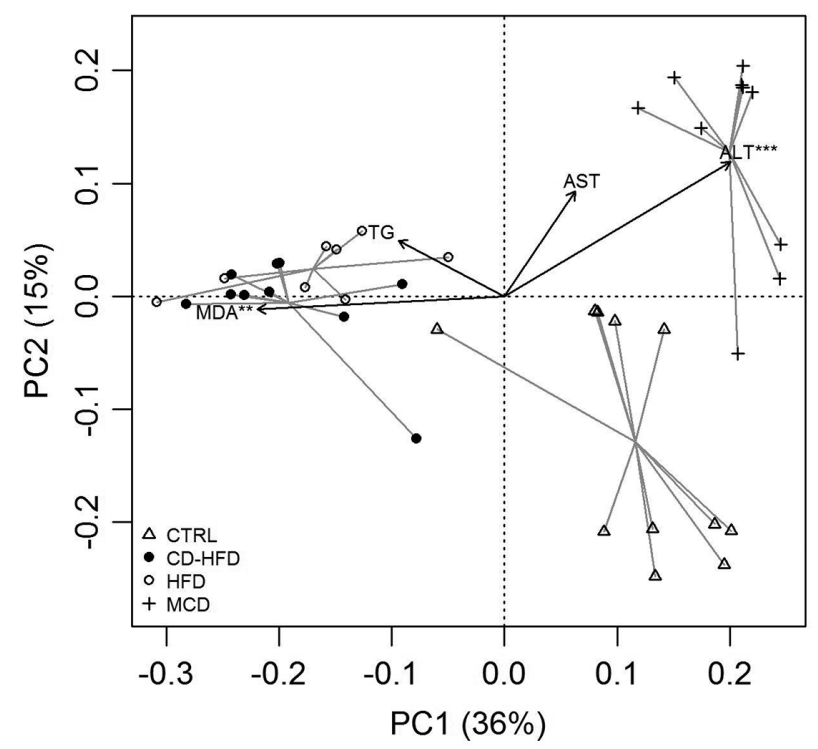

Abstract IDDF2018-ABS-0162 Figure 1 PCA ordination of the gut microbiota communities, fitted with serum and liver tissue biochemistry assays $\left({ }^{* *} \mathrm{P}<0.001,{ }^{* *} \mathrm{P}<0.01\right)$

Results Histological scores showed that MCD induced the severest steatohepatitis, followed by $\mathrm{HF}$ and CD-HF diets. Based on operational taxonomic units (OTUs) at cutoff of 97\% similarity, there were significant (PERMANOVA, $\mathrm{p}=0.001$ ) differences in overall gut bacterial communities among MCD, HF, CD-HFD, and the Control, forming three major clusters in PCA ordination with HF and CD-HF groups more similar (figure 1). Furthermore, $\alpha$-diversity of $\mathrm{HF}$ and CD-HF groups, including observed OTU numbers, Shannon index, and Pielou evenness were significantly (ANOVA, $\mathrm{p}<0.05)$ higher than the Control and MCD group. Overall, 